\title{
Distribución de la fuerza de trabajo en enfermería en la Región de las Américas
}

\author{
Silvia Helena De Bortoli Cassiani, ${ }^{1}$ Maria Cristina Hoyos, ${ }^{1}$ Maynara Fernanda \\ Carvalho Barreto, ${ }^{1}$ Katie Sives ${ }^{1}$ y Fernando Antonio Menezes da Silva ${ }^{1}$
}

Forma de citar

Cassiani SHB, Hoyos MC, Barreto MFC, Sives K, da Silva FAM. Distribución de la fuerza de trabajo en enfermería en la Región de las Américas. Rev Panam Salud Publica. 2018;42:e72. https://doi org/10.26633/RPSP.2018.72

RESUMEN Objetivo. Describir la distribución de la fuerza de trabajo de enfermería en países de la Región de las Américas y relacionar el número de recursos humanos en enfermería con las tasas de mortalidad materna.

Métodos. Análisis descriptivo y exploratorio de 27 países de la Región. Las variables del estudio fueron la proporción de profesionales por país y subregión, la categoría profesional y la razón de enfermero-médico. Se utilizó la proporción de mortalidad materna, que es un indicador general de salud de la población, para analizar la relación con el cuantitativo de los enfermeros. Se analizaron la distribución de frecuencias y la densidad del recurso humano de enfermería por país y subregión.

Resultados. La distribución de enfermería es heterogénea. Existen países con más de 80 enfermeros por 10000 habitantes y otros con menos de cinco profesionales por 10000 habitantes. En 34,1\% de los países, la relación enfermero-médico es menor a uno. Se observan diferencias en la distribución de personal de enfermería por región, subregión y al interior de país.

Conclusiones. En varios países, el número de enfermeros por habitantes es menor a lo esperado. La mayoría de los países muestran un retraso importante en la relación del número de enfermeros licenciados respecto al personal técnico y auxiliar. Es necesario implementar iniciativas para aumentar el número de enfermeros licenciados en toda la Región.

Palabras clave Fuerza de trabajo; recursos humanos; personal de enfermería; Américas.

La Organización Mundial de la Salud (OMS) y la Organización Panamericana de la Salud (OPS) consideran la fuerza de trabajo en salud como uno de los principales componentes en la consecución de los Objetivos de Desarrollo Sostenible (ODS) y de la estrategia del acceso y cobertura universal de salud $(1,2)$.

Es necesario que la distribución de los recursos humanos sea adecuada, que

\footnotetext{
Organización Panamericana de la Salud, Washington D.C., Estados Unidos de América. Enviar la correspondencia a Silvia Cassiani: cassianis@paho.org
}

exista un número de profesionales de la salud activos suficiente y con las competencias necesarias para fortalecer los sistemas de salud y expandir la atención primaria, y contribuir así a que los países alcancen la cobertura universal de salud $(3,4)$.

Las estadísticas de los últimos cinco años muestran un déficit mundial de 12,9 millones de profesionales (4-6) de los cuales, según la OMS, aproximadamente 4,3 millones corresponden a médicos y enfermeros $(7,8)$.

Los cambios en la dinámica poblacional y demográfica, el envejecimiento de la población y el aumento de las enfermedades crónicas son factores que impactan en la fuerza de trabajo (9).

En la mayoría de los países, la concentración de profesionales en áreas urbanas y en los servicios de atención terciaria de salud resultan en una fragilidad en la atención primaria a la salud (APS) y puede estar relacionada con la mala distribución de la fuerza de trabajo y la migración de los recursos humanos en salud $(8,10)$ que afectan, sobre todo, a las regiones más pobres y remotas $(9,11)$.

Ante esta situación, se han puesto en marcha estrategias que inciten a los

Este es un artículo de acceso abierto distribuido bajo los términos de la licencia Creative Commons Attribution-NonCommercial-NoDerivs 3.0 IGO, que permite su uso, distribución y reproducción en cualquier medio, siempre que el trabajo original se cite de la manera adecuada. No se permiten modificaciones a los articulos ni su uso comercial. Al reproducir un articulo no debe haber ningún indicio de que la OPS o el artículo avalan a una organización un producto específico. El uso del logo de la OPS no está permitido. Esta leyenda debe conservarse, junto con la URL original del artículo.

Este manuscrito ha sido corregido: https://doi.org/10.26633/RPSP.2019.319 
gobiernos y a las organizaciones a mejorar las condiciones laborales de los recursos humanos para la salud, a capacitarlos y retenerlos de manera eficaz y a disponer del número adecuado de personal $(4,12)$.

La OPS/OMS busca ampliar el acceso equitativo al personal sanitario, fortalecer la rectoría y la gobernanza, aumentar y mejorar el financiamiento con equidad y eficiencia (13) y potenciar y guiar las políticas nacionales de recursos humanos de los países de la Región, con el fin de subsanar la escasez de personal sanitario y mejorar su distribución geográfica (14). Insta a los países a planificar el personal de salud y a establecer mecanismos para contener la migración y la mala distribución de estos profesionales (15).

La distribución adecuada de la fuerza de trabajo es fundamental para el avance de la salud universal y la atención segura y de calidad. El número y la calidad de los profesionales presentan una asociación positiva con la cobertura de la inmunización, al alcance de la atención primaria, y con la supervivencia materna e infantil $(7,8)$.

En términos cuantitativos, un aumento de $10 \%$ de la fuerza de trabajo en salud se relaciona con una disminución de aproximadamente $5 \%$ en la mortalidad materna. La literatura científica evidencia que un incremento en la distribución y densidad de los recursos humanos y el fortalecimiento de los sistemas de salud favorecen la disminución de la tasa de mortalidad materna (8). Estudios recientes (16-18) han explorado esta asociación, tomaron los indicadores que muestran fortaleza de los sistemas de salud y desarrollaron análisis exploratorios entre desenlaces de salud pública como mortalidad materna y los indicadores potenciales de fortalecimiento de sistemas de salud y la densidad del recurso humano en salud.

En este sentido, si se tiene en cuenta que la enfermería representa la mayor categoría en la fuerza de trabajo en salud, es necesario estimar el número de sus profesionales para apoyar las decisiones y la gestión de los servicios, y alcanzar la cobertura de salud universal (19-21).

El personal de enfermería de la Región de las Américas está conformado por profesionales según su formación: a) enfermeros licenciados, b) tecnólogos, técnicos de enfermería, y c) auxiliares y asistentes básicos de enfermería.
Los enfermeros licenciados también conocidos como enfermeros registrados $\mathrm{o}$, simplemente, enfermeros, tienen una formación universitaria de cuatro o cinco años. Los tecnólogos de enfermería tienen una educación secundaria y hasta tres años de formación profesional o educación técnica. En algunos países, se los denomina como técnicos de nivel superior, en cuyo caso realizan tres años de formación universitaria. Los técnicos de enfermería tienen de dieciocho meses hasta tres años de formación de nivel medio, y los auxiliares o asistentes básicos de enfermería poseen una educación básica de doce a dieciocho meses de capacitación formal, con una carga horaria de 900 a 1800 horas de curso. El tiempo de formación está relacionado con las responsabilidades y las competencias técnico-científicas de cada categoría profesional en la prestación de los cuidados de salud.

Los objetivos del presente estudio son describir la distribución de la fuerza de trabajo de enfermería en la Región de las Américas, en términos cuantitativos y según la formación profesional, y relacionar el número de enfermeros con las tasas de mortalidad materna por países de la Región.

\section{MATERIALES Y MÉTODOS}

Se realizó un análisis descriptivo y exploratorio de 27 países de la Región de las Américas, con los datos numéricos disponibles acerca de la fuerza de trabajo de enfermería. Las variables del estudio fueron: a) la proporción de profesionales por país y subregión (número de enfermeros por cada 10000 habitantes en una población, para un año dado, en un determinado país o territorio), b) la categoría profesional, y c) la razón enfermero-médico (proporción de enfermeros dividida por la proporción de médicos).

En cuanto a la fuente de datos, la proporción de enfermeros por cada 10000 habitantes (hab) como un indicador general del nivel de salud de la población, la razón enfermero-médico, y la proporción de mortalidad materna por 100000 nacidos vivos se obtuvieron a través de la Plataforma de Información en Salud de las Américas (PLISA) (22) de la OPS/ OMS que mantiene un conjunto de datos públicos agregados en la plataforma.
Para conocer la distribución por categoría profesional, se realizaron una serie de seminarios virtuales, sobre la "Contribución de la enfermería en la Región de las Américas y su potencial" presentados por enfermeros pertenecientes a los diferentes ministerios de salud de los países $\mathrm{y}$ asociaciones de enfermería o universidades. En estos seminarios, se recolectaron datos de 27 (77\%) de los 33 países de la Región. El Programa de Enfermería y Técnicos en Salud de la OPS/OMS organizó los encuentros, que se transmitieron en modalidad virtual por las salas WEBEX entre los años 2015 y 2017. Asimismo, se utilizaron datos de la fuerza de trabajo en enfermería disponibles en las páginas virtuales oficiales de los ministerios de salud o de otras organizaciones gubernamentales. La recolección de esta información se realizó a través de una base de datos, donde se consignaron las variables proporcionadas por país. Todos los registros fueron manejados con criterios de confidencialidad y seguridad. La información consignada fue verificada, se utilizaron filtros y se ingresaron los datos por doble entrada.

Se calcularon las medianas por región y se compararon las diferencias entre las medianas de los grupos. Se utilizó la prueba de Kruskal-Wallis para comparar si estas diferencias eran significativas $(P<0,05)$. Para el procesamiento de los datos y la construcción del cuadro y las figuras, se utilizaron los programas Ex$\mathrm{cel}^{\circledR}$, ArcGis $10^{\circledR}$, Statistical Package for the Social Sciences (SPSS) versión $21^{\circledR}$ y Data Analysis and Statistical Software (STATA) versión $12^{\circledR}$.

El estudio atendió las normas internacionales de ética en investigación. Al tratarse de un análisis de datos secundarios, no fue necesario someterlo al Comité de Revisión Ética de la OPS/OMS.

\section{RESULTADOS}

De los 27 países incluidos, la cantidad total del personal de enfermería fue de 9655748 profesionales, hasta octubre de 2017. De estos, 47,1\% ( $n=4545$ 509) son enfermeros licenciados; $27 \%$ ( $n=2$ 606 551) son tecnólogos y técnicos, y $25,9 \%(n=2503688)$ son auxiliares de enfermería.

En el cuadro 1 se presenta la razón enfermero-médico y la densidad de profesionales agrupados por país, subregión y 
CUADRO 1. Densidad de personal de enfermería y razón enfermero-médico en la Región de las Américas, 2017

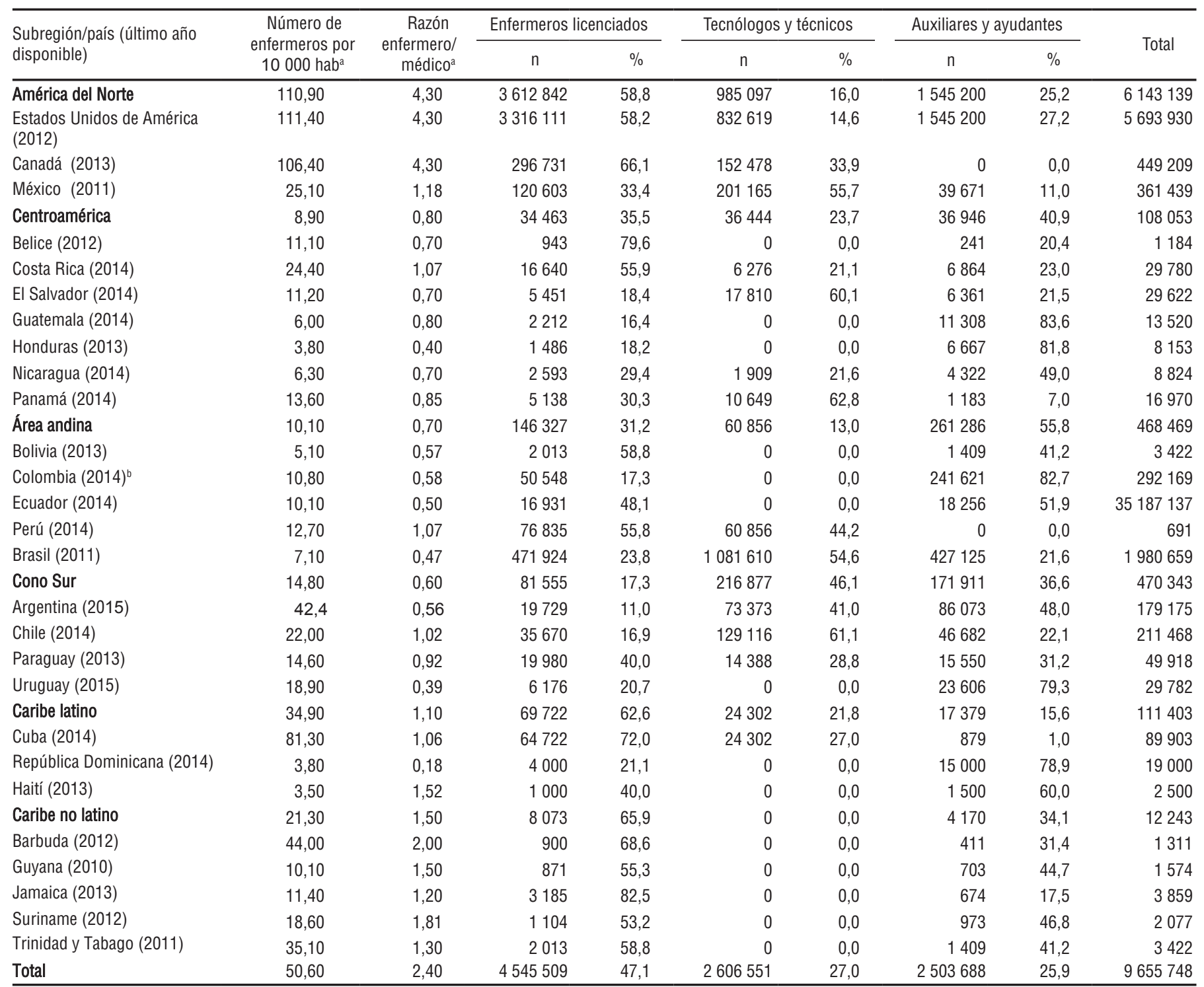

${ }^{a}$ Enfermeros: en ese caso específico, son considerados los enfermeros registrados o licenciados y técnicos de enfermería tal como se presentan en la Plataforma de Información en Salud de las Américas (PLISA) de la OPS/OMS.

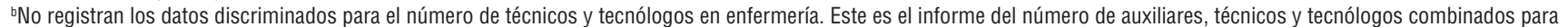

Colombia.

Fuentes: Plataforma Plisa (22); páginas virtuales oficiales de los ministerios de salud y otras organizaciones gubernamentales (23-31).

categoría profesional. Los datos están divididos en ocho subregiones: América del Norte, México, Centroamérica, Área andina, Brasil, Cono Sur, Caribe latino y Caribe no latino. Debido a su tamaño poblacional, Brasil y México se analizaron por separado, tal como lo organiza la OPS/OMS en su publicación sobre los indicadores básicos de la situación de salud en las Américas (22).
El número de enfermeros (licenciados o registrados y técnicos de enfermería) por 10000 hab varía desde 3,5 (Haití) hasta 111,4 (Estados Unidos de América, EEUU) y la mediana es de 10,4 enfermeros por 10000 hab. En el cuadro 1 se observa que, en la mitad de los países, el índice de enfermeros por 10000 hab es menor o igual a 10,4. No obstante, existe una variación amplia y cabe considerar que EEUU (111,4), Canadá $(106,2)$ y Cuba $(81,3)$ tienen la mayor proporción de enfermeros por 10000 hab.

Los países de la subregión Centroamérica tienen 14 enfermeros o menos por 10000 hab, con excepción de Costa Rica donde hay 24 enfermeros por 10000 hab. En la subregión andina y el Cono Sur, la proporción es de alrededor de 20 por 10000 hab, y al menos la mitad de los 
países ubicados en ambas zonas están por debajo de 10 por 10000 hab.

Para el caso del Caribe no latino, se observa una mayor distribución y una más amplia proporción de enfermeros licenciados en comparación con los otros profesionales. Por otra parte, se observa una distribución similar a las otras subregiones, con excepción de Cuba, que cuenta con 81,3 enfermeros por cada 10000 hab, de los cuales $72 \%$ son licenciados.

América del Norte, con Canadá y EEUU, muestra la densidad más alta de enfermeros, y más de $60 \%$ de la fuerza de trabajo en enfermería corresponde a enfermeros licenciados o registrados.

En cuanto a la relación enfermeromédico, 55,6\% $(n=15)$ de los países presentan menos de un enfermero por médico; 37\% $(n=10)$ de uno hasta tres enfermeros por médico, y $7,4 \%(n=2)$ tres o más enfermeros por médico. Esta relación varía entre las subregiones y al interior de las ellas. En América del Norte, la relación es de 4,3 enfermeros por médico. En los países de la subregión andina y el Cono Sur, hay más médicos que enfermeros. En los países de América del Norte y del Caribe no latino, el número de enfermeros supera al de los médicos. En las demás subregiones, existen más médicos que enfermeros.

En la figura 1 se muestran las medianas de la densidad de enfermeros por subregión. El Caribe latino muestra una variabilidad mayor, con una mediana de 61,2 enfermeros por 10000 hab y rangos que varían desde 3,5 enfermeros en Haití, hasta 81,7 y 81,3 enfermeros por 10000 hab en Cuba y Martinica, respectivamente. La mediana de la subregión andina es de 10,1 enfermeros por 10000 hab y la del Cono Sur, de 16,7. El Caribe no latino tiene una densidad de 33,6 enfermeros por 10000 habitantes.

Las diferencias entre medianas de los países agrupados por región presentaron una heterogeneidad en la densidad de enfermeros por 10000 hab $(P=0,002)$.

En cuanto a la cantidad de enfermeros licenciados, Canadá, EEUU y Cuba suman 80,9\% del total de la Región. De esta manera, tres países de la Región concentran la mayoría de los enfermeros licenciados.

En la mayoría de los países, el número de tecnólogos, técnicos y auxiliares supera al de los enfermeros licenciados, lo cual muestra que hay más inversión en la formación y mercado laboral para profesionales de esos niveles (figura 2).

Es evidente la diversidad, donde destaca que países del Caribe latino y del Caribe no latino tienen la mayor proporción de enfermeros licenciados respecto a las demás categorías de enfermería, con excepción de la República Dominicana donde, junto con Argentina, Chile, Colombia, El Salvador, Guatemala, Honduras, solo alrededor de $20 \%$ de la fuerza laboral en enfermería conformada por enfermeros licenciados. Belice, Cuba y Jamaica, por el contrario, tienen la mayor proporción de enfermeros licenciados con respecto a las demás categorías de personal de enfermería.

En la Región se observa una distribución desigual del personal de enfermería por país y subregión. Al interior de los países existen diferencias en la densidad de los profesionales, es decir, coexisten zonas o áreas con mayor número de personal de enfermería, con otras caracterizadas por un bajo número.

Para ejemplificar la diferencia de la densidad de personal de enfermería dentro de un país, se realizó un análisis con datos desagregados por estado y por categoría profesional de un país (figura 3). Brasil fue seleccionado por poseer el mayor número de escuelas de enfermería en la Región, con datos completos y actualizados por estado, lo cual facilitaba el análisis.

Los datos muestran un total de 1998 109 profesionales de enfermería, de los cuales $476754(23,8 \%)$ son enfermeros licenciados, 1094091 (54,8\%) son técnicos de enfermería y 426933 (21,4\%) son auxiliares de enfermería.

La proporción de enfermeros licenciados por 10000 hab en los estados varía de 39,9 (Distrito Federal, DF) hasta 12,7 (Pará, PA). La relación de licenciados con respecto a otros profesionales también es desigual y en la mayoría de los estados se encuentra una proporción de dos licenciados por cada ocho técnicos o auxiliares de enfermería.

La variación de los técnicos de enfermería es de 112,1 (Amapá, AP) hasta 33,9 técnicos por 10000 hab (Alagoas, AL). En relación con los auxiliares de enfermería, la variación es de 42,7 en São Paulo (SP), hasta 6 en el estado de Maranhão (MA). La discrepancia entre el número de enfermeros por estado pone

\section{FIGURA 1. Medianas, rango intercuartílico de la densidad de enfermeros por cada 10000 hab por subregión}

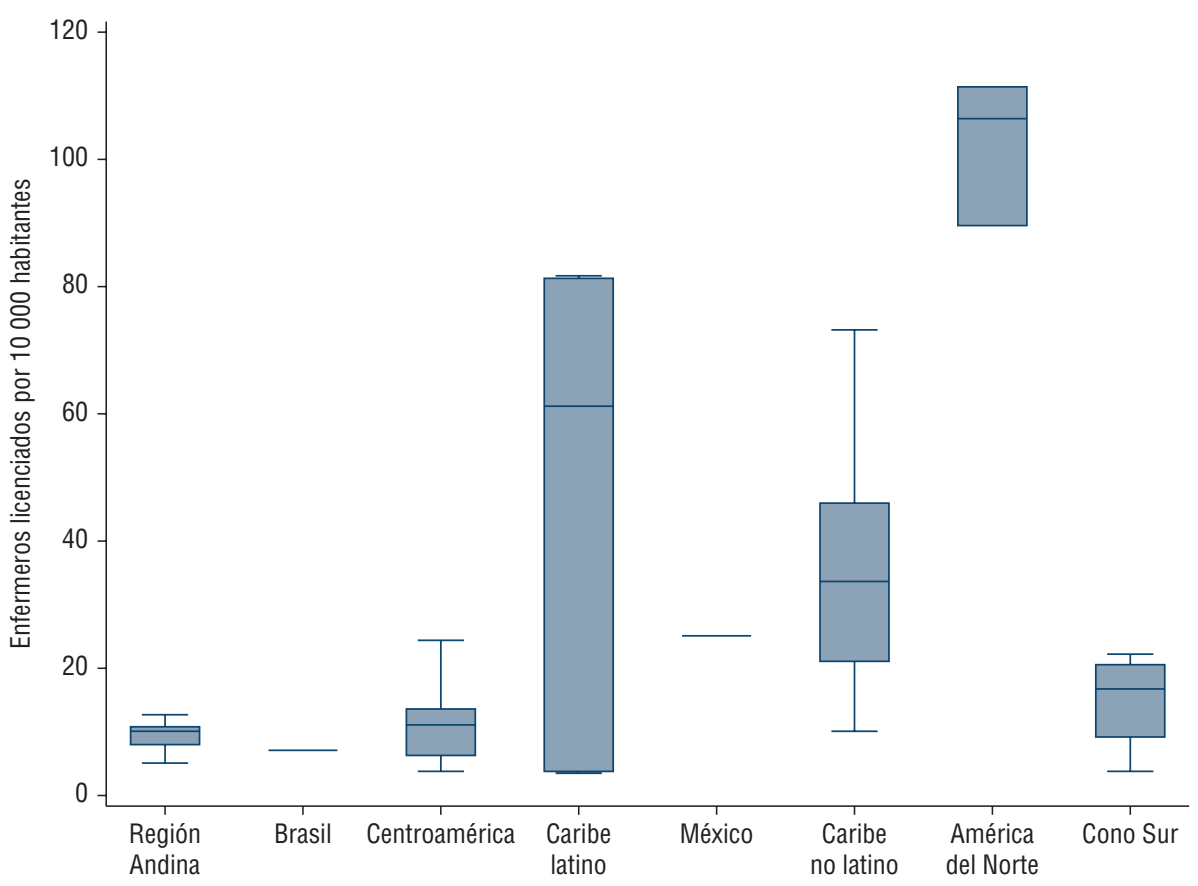

Fuentes: Plataforma Plisa (22); páginas virtuales oficiales de los ministerios de salud y otras organizaciones gubernamentales (23-31). 
FIGURA 2. Proporción de enfermeros licenciados, tecnólogos técnicos y auxiliares de enfermería por país y subregión

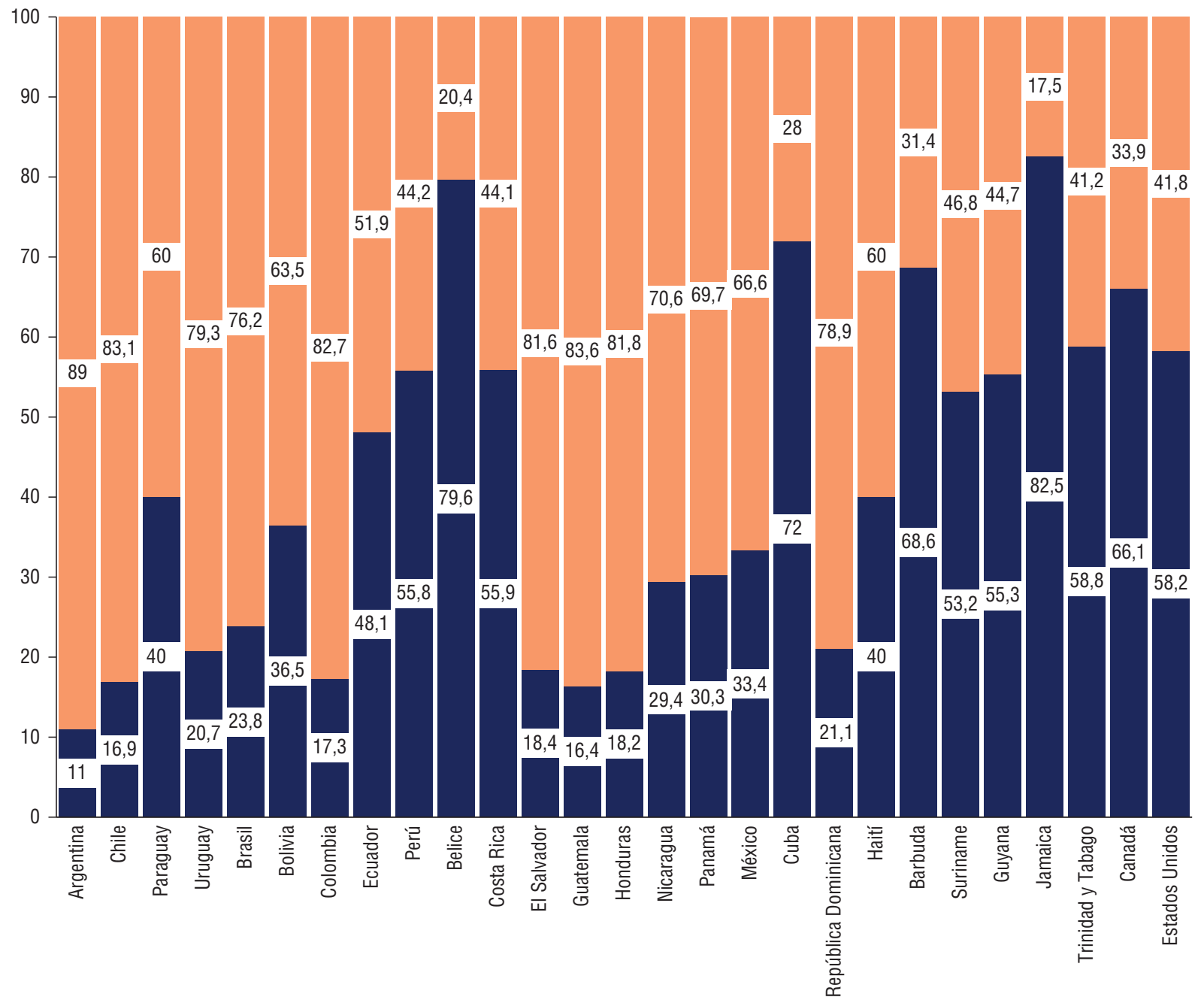

Proporción otro profesional de enfermería — Proporción enfermeros licenciados

Fuentes: Plataforma Plisa (22); páginas virtuales oficiales de los Ministerios de Salud y otras organizaciones gubernamentales (23-31).

de manifiesto la desigualdad numérica y el déficit de profesionales para atender la demanda de la población y los servicios de salud con la calidad y la seguridad necesarias.

En cuanto a la relación entre la densidad de enfermeros por habitantes y el índice de mortalidad materna, (figura 4) se observa que EEUU y Canadá, con más de 100 enfermeros por 10000 hab, presentan una mortalidad de cinco mujeres por cada 100000 nacidos vivos, contrastando con Bolivia, Honduras y Nicaragua con menos de 10 enfermeros por cada 10000 hab, con una tasa superior a 100 por cada 100000 nacidos vivos. El análisis exploratorio mostró que los países con mayor densidad de enfermeros por población presentaron menor índice de mortalidad materna.

\section{DISCUSIÓN}

Los países de la Región de las Américas analizados en ese estudio presentan un patrón de distribución heterogéneo con respecto a la densidad de profesionales de la salud, con rangos que varían desde 3,5 enfermeros por 10000 hab en Haití, hasta más de 100 en EEUU y Canadá.

Las diferencias entre la densidad del personal en los países y al interior de cada uno de ellos se deben al mercado laboral, a la oferta de programas de formación de enfermeros y los incentivos financieros y no financieros ofertados entre las regiones, ciudades y estados. La inversión que hacen algunos países para contratar auxiliares de enfermería se origina en las políticas nacionales, ya que se trata de un profesional que recibe un sueldo menor, con una formación más limitada y con menor calificación profesional que los demás (32).

\footnotetext{
Acre (AC); Alagoas (AL); Amapá (AP); Amazonas (AM); Bahia (BA); Ceará (CE); Distrito Federal (DF); Espirito Santo (ES); Goiás (GO); Maranhão (MA); Mato Grosso (MT); Mato Grosso do Sul (MS); Minas Gerais (MG); Pará (PA); Paraíba (PB); Paraná (PR); Pernambuco (PE); Piauí (PI); Rio de Janeiro (RJ); Rio Grande do Norte (RN); Rio Grande do Sul (RS); Rondônia (RO); Roraima (RR); Santa Catarina (SC); São Paulo (SP); Sergipe (SE); Tocantins (TO).
} 
FIGURA 3. Densidad de recurso humano en enfermería de Brasil, por estado, 2017

a) Número de enfermeros licenciados por 10000 habitantes

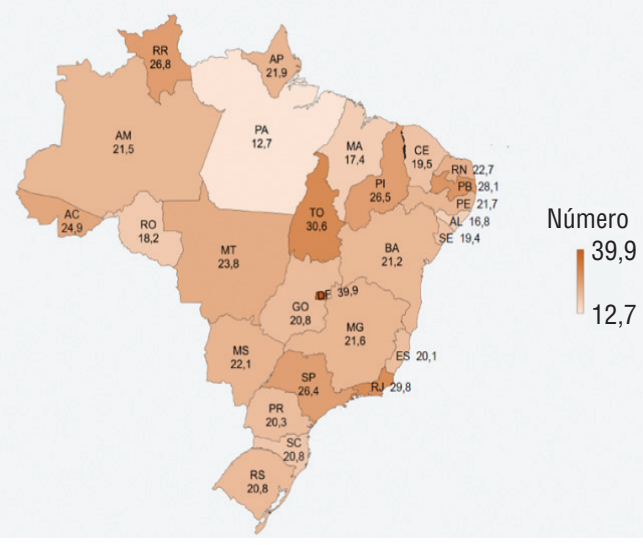

c) Número de auxiliaries de enfermería por 10000 habitantes

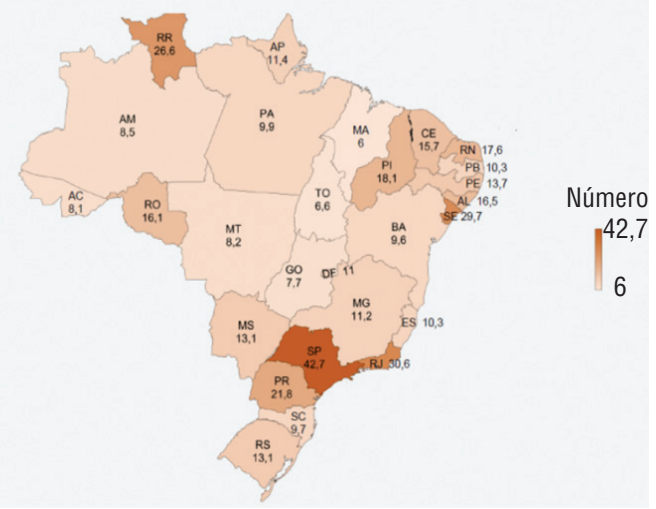

b) Número de técnicos de enfermería por 10000 habitantes

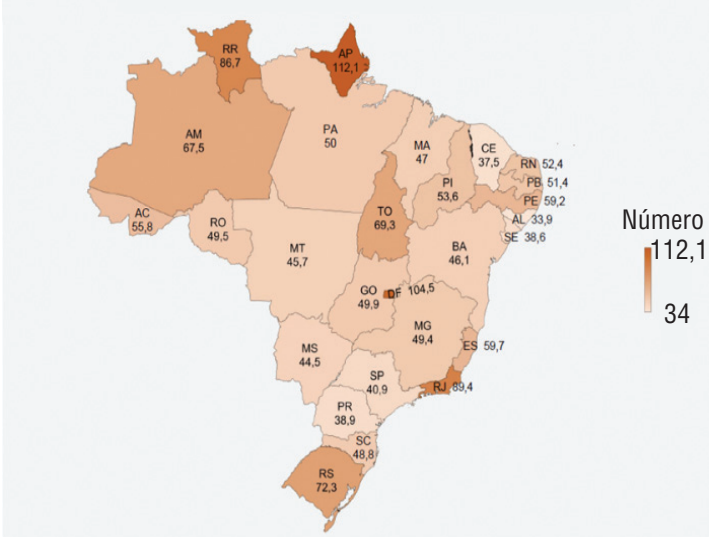

d) Número de profesionales de enfermería por 10000 habitantes

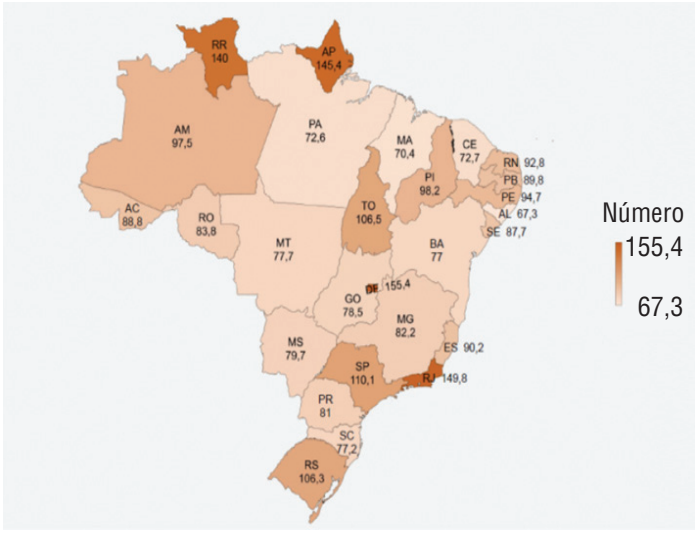

Fuente: Conselho Federal de Enfermagem (COFEN), 2017 (28). ${ }^{2}$

Al comparar los países de la Región con aquellos que conforman la Organización para la Cooperación y el Desarrollo Económicos (OECD), se observa que EEUU y Canadá poseen una tendencia similar a la de Alemania, Australia, Dinamarca, Finlandia, Japón, Noruega y Suiza, donde existen entre $100 \mathrm{y}$ 170 enfermeros por 10000 hab. Cuba y algunas islas del Caribe presentan una proporción similar a la del Reino Unido y Nueva Zelanda, con un rango de entre 80 y 100 enfermeros licenciados por 10000 hab. Barbuda, Costa Rica, Chile, México, Suriname, Trinidad y Tabago y Uruguay muestran una tendencia similar a la de Turquía, con un rango de 19 a 40 enfermeros por 10000 habitantes. En Argentina, Belice, Bolivia, Brasil, Colombia, Ecuador, El Salvador, Guatemala, Guyana, Haití, Honduras, Jamaica, Nicaragua, Panamá, Paraguay y República Dominicana, se encontró una proporción inferior a los números de otros países como Corea del Sur, España e Israel, que poseen un rango de 40 a 60 enfermeros por 10000 habitantes (33).

En la mayoría de los países de la Región, aproximadamente $70 \%$ de la fuerza de trabajo de enfermería está conformada por tecnólogos, técnicos y auxiliares de enfermería. En algunos países, la relación es de dos enfermeros licenciados por cada ocho técnicos, tecnólogos y auxiliares de enfermería. Es clara la necesidad de aumentar la proporción de los enfermeros licenciados respecto al resto del recurso humano en enfermería en la Región, para fortalecer los servicios, la calidad de la atención a la salud y la seguridad de los pacientes $(34,35)$.

Se observa que la distribución del personal médico y de enfermeros varía según el país: Argentina, Cuba, Islas Caimán y Uruguay tienen la mayor proporción de médicos por población, con rangos de 40 a 70 por cada 10000 hab. En la región de África, la relación es de ocho enfermeros por cada médico. $\mathrm{Al}$ comparar con otras regiones, se observa que en el Pacífico Occidental la proporción es de 1,5 enfermeros por cada médico (36). Cabe recordar que, según las metas regionales de recursos humanos para salud y los desafíos de Toronto (2006-2015), la meta propuesta por la OPS/OMS para la razón enfermero-médico es de, al menos, un enfermero por cada médico $(37,38)$. Quince países (34\%) de la Región aún no cumplen esa meta.

El análisis exploratorio de este estudio sobre la mortalidad materna y el número de enfermeros son congruentes con la literatura científica. La OMS ha presentado relación entre la densidad de los trabajadores de salud y su impacto en la reducción de la mortalidad materna 
FIGURA 4. Enfermeros por 10000 habitantes e índice de mortalidad materna por cada 100000 nacidos vivos

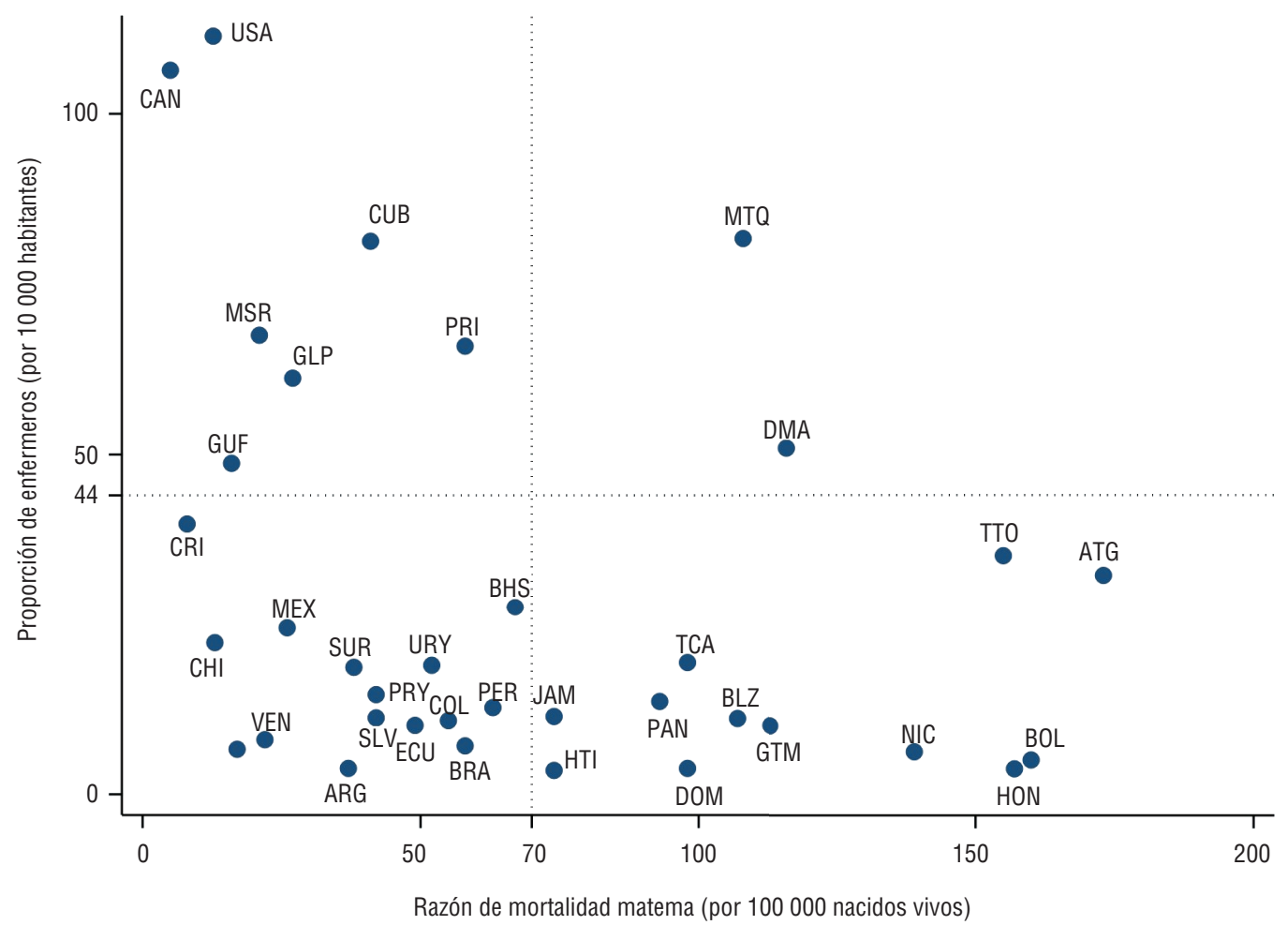

Fuentes: Plataforma Plisa (22), páginas virtuales oficiales de los Ministerios de salud y otras organizaciones gubernamentales (23-31).

$(7,8,16,17)$. Además, estudios realizados en EEU y en algunos países de Europa mostraron que, a medida que el número de enfermeros licenciados aumenta, hay una disminución de la mortalidad hospitalaria $(39,40)$.

En los últimos años, los países de ingresos económicos altos han impulsado la educación y la capacitación profesional en enfermería para atender la creciente demanda por profesionales (33). Incentivar a los jóvenes, acreditar las escuelas, fortalecer e impulsar a la profesionalización $\mathrm{y}$, finalmente, crear mercados laborales atractivos son estrategias que pueden impactar de manera positiva en el aumento de la densidad de enfermeros licenciados y fortalecer los servicios de salud.

Por otra parte, la distribución heterogénea, las dificultades para atraer profesionales a las áreas remotas y rurales y las inequidades que prevalecen en las condiciones laborales y en los países afectan la formación de recursos humanos en enfermería y el acceso a profesionales calificados en los servicios de salud. A lo anterior, cabe añadir que la migración, sobre todo en países del
Caribe no latino, es una realidad que impacta en el aspecto cuantitativo del personal de enfermería y los servicios de salud (41). Para retenerlos, es necesario crear incentivos y mejorar sus condiciones de trabajo. El establecimiento de acuerdos y registros entre los países de origen y de destino de los profesionales, la mejora de los sistemas de datos y el monitoreo eficaz de esos flujos migratorios, también son imperativos.

El análisis presentado permitirá avanzar hacia las políticas y la construcción del conocimiento de los recursos humanos en enfermería en la Región. Sin embargo, se sugieren estudios adicionales que analicen y estimen, con un nivel de desagregación de los datos, la distribución geográfica, el nivel de atención en salud, la composición del recurso y el perfil del personal de enfermería. La caracterización de estos atributos permitirá la construcción de un perfil fundamental para una planificación estratégica y gestión del recurso humano.

Las limitaciones de este estudio comprenden la falta de datos de algunos países y las diferencias del período de registro de datos notificados. Esta consideración debe tenerse en cuenta al interpretar los resultados. Los reportes de la fuerza de trabajo en salud han descrito limitaciones para la comparabilidad: las directrices sugeridas por la OMS no siempre son aplicadas, pues algunos países carecen de la capacidad técnica para hacer un seguimiento preciso del personal sanitario (42). No obstante, esta exploración de los datos permite conocer la situación de la enfermería en la Región de manera general. Por otra parte, es importante mantener y fortalecer los sistemas de información de los países que permitan actualizar los datos sobre los recursos humanos para la salud.

La exploración entre la densidad de enfermeros por población y la mortalidad materna, no incluyó un análisis de correlación ni ajuste por otras variables, lo cual limita una conclusión para asociar estos dos indicadores.

\section{CONCLUSIONES}

La distribución laboral de los profesionales de enfermería es heterogénea. Al interior de las regiones se observa una tendencia similar, pero la 
diferencia entre regiones varía ampliamente, lo que pone en evidencia la desigualdad en la distribución de la fuerza de trabajo en la región de las Américas.

En varios países, el número de enfermeros licenciados por habitantes es menor a lo esperado. Por otra parte, la mayoría de los países también presentan un retraso importante en la proporción de enfermeros licenciados respecto al personal técnico y auxiliar. Es necesario implementar iniciativas dirigidas a aumentar el número de enfermeros licenciados y fortalecer su formación en la Región.
Los países deben definir políticas que marquen el futuro de la enfermería. La estimación del recurso humano es fundamental para orientar la planificación y la toma de decisiones en todos los niveles de la atención de salud.

El fortalecimiento de los sistemas de información, registros e indicadores nacionales que permitan tener actualizadas las bases de datos de recursos humanos para la salud pueden contribuir a la planificación de programas y políticas públicas, orientando a los ministerios de salud y a los demás sectores al trazar sus planes estratégicos

\section{REFERENCIAS}

1. Organización Mundial de la Salud (OMS). Estrategia mundial de recursos humanos para la salud: personal sanitario 2030 [Internet]. 69. ${ }^{a}$ Asamblea Mundial de la Salud. 20 -28 mayo de 2016. Ginebra: OMS; 2016 (resolución WHA69.19). Disponible en: http://apps.who.int/gb/ ebwha/pdf_files/WHA69/A69_R19-sp. pdf Acceso el 20 de setiembre de 2017.

2. Organización Mundial de la Salud. Estrategia para el acceso universal a la salud y la cobertura universal de salud. 53. Consejo Directivo. 66. ${ }^{a}$ Sesión del comité regional de la OMS para las Americas. 29 de setiembre al 3 de octubre de 2014. Washington, D.C. Disponible en: http:// www.paho.org $/ \mathrm{hq} /$ index.php?option= com_content\&view $=$ article $\& i d=9774 \%$ 3A2014-53rd-directing-council\&catid= $8811 \% 3$ Adc-documents\&Itemid $=410$ 62\&lang=es Acceso el 20 de octubre de 2017.

3. World Health Organization (WHO). Global strategy on human resources for health: Workforce 2030. Geneva: WHO; 2016. Disponible en: http://apps.who. int/iris/bitstream/10665/250368/1/9789 241511131-eng.pdf Acceso el 20 de setiembre de 2017.

4. World Health Organization. High-level Comission on Health Employment and Economic Growth. Working for Health and Growth: Investing in the health workforce. 2016. Disponible en: http://apps. who.int/iris/bitstream/10665/250047/1/ 9789241511308-eng.pdf?ua=1 Acceso el 20 de setiembre de 2017.

5. Organización Mundial de la Salud (OMS). Una verdad universal: No hay salud sin agentes sanitarios. Alianza mundial en pro del personal sanitario, Organización Mundial de la Salud. Noviembre 2013. Disponible en: http://www.who.int/ workforcealliance/knowledge/resources/hrhreport2013/es/ Acceso el 20 de setiembre de 2017.
6. World Health Organization (WHO). Health Workforce 2030: towards a global strategy on human resources for health. Geneva: WHO; 2015.

7. Organización Mundial de la Salud (OMS) Informe sobre la salud en el mundo, Colaboremos por la salud 2006: 227. Disponible en: http://www.who.int/ whr/2006/es/ Acceso el 20 de setiembre de 2017.

8. Global Equity Initiative. Harvard University. Human Resources for Health: Overcoming the crisis. Joint Learning Initiative; 2004:217. Disponible en: http:/ / www.who.int/hrh/documents/JLi_hrh_ report.pdf Acceso el 10 de setiembre de 2017.

9. Portela GZ, Fehn AC, Ungerer RLS, Poz MRD. Recursos humanos em saúde: crise global e cooperação internacional. Cienc Saude Coletiva. 2017;22(7):2237-46. Disponible en: http://www.scielo.br/scielo. php? script $=$ sci_arttext \& pid $=$ S1413$81232017002702237 \& \operatorname{lng}=$ en Acceso el 10 de octubre de 2017.

10. Crisp N, Chen L. Global supply of health professionals. N Engl J Med. 2014;370(23): 2247-8. Disponible en: http://www.ncbi. nlm.nih.gov/pubmed/24897096 Acceso el

11. Girardi SN, Carvalho CL, Wan der Maas L, Araujo JF, Massote AW, Stralen ACSV. Preferências para o trabalho na atenção primária por estudantes de medicina em Minas Gerais, Brasil: evidências de um experimento de preferência declarada. Cad Saude Publica. 2017;33(8):e00075316. Disponible en: http://www.scielo.br/scielo. php? script=sci_arttext\&pid= S0102-311X2017000805012\&lng=en. http:/ / dx.doi.org/10.1590/0102311x00075316 Acceso el 22 de setiembre de 2017.

12. Organización Mundial de la Salud. Comisión de las Naciones Unidas: Invertir en el cuerpo mundial de profesionales 10 de octubre de 2017.
El trabajo colaborativo entre organizaciones internacionales, gobiernos, asociaciones gremiales y universidades puede, por medio del análisis, planificar y cambiar, al menos en términos cuantitativos, el futuro de la enfermería en los países de la región de las Américas.

Conflicto de intereses. Ninguno declarado por los autores.

Declaración. Las opiniones expresadas en este manuscrito son responsabilidad de los autores y no reflejan necesariamente los criterios ni la política de la RPSP/PAJPH o de la OPS sanitarios permitirá la creación de empleo e impulsará el crecimiento económico. Comisión las Naciones Unidas sobre el Mercado Laboral, 2016. Disponible en: http: / / www.who.int/hrh/com-heeg / ComHEEG_PR_SP.pdf?ua=1 Acceso el 20 de julio de 2017 .

13. Campbell J, Dussault G, Buchan J, PozoMartin F, Guerra AM, Leone C, et al. A universal truth: no health without a workforce. Forum Report. Third Global Forum on Human Resources for Health. Recife, Brazil. Geneva: Global Health Workforce Alliance and World Health Organization; 2013.

14. Organización Mundial de Salud/ Organización Panamericana de Salud. Estrategia de recursos humanos para el acceso universal a la salud y la cobertura universal de salud. 2017. Disponible en: http://www.paho.org/hq/index.php? option=com_content\&view $=$ article\&i$\mathrm{d}=2558 \% 3$ Ahuman-resources-health\&cat$\mathrm{id}=526 \% 3 \mathrm{Ainfo} \&$ Itemid $=233 \&$ lang $=$ es Acceso el 10 de octubre de 2017.

15. World Health Organization. Stregthening Health Systems to improve Health Outcomes. 2007:56. Disponible en: http:/ / apps.who.int/iris / bitstream / 10665 / 43918/1/9789241596077_eng.pdf Acceso el 20 de julio de 2017.

16. Van Lerberghe W, Matthews Z, Achadi E, Ancona C, Campbell J, Channon A, et al. Country experience with strengthening of health systems and deployment of midwives in countries with high maternal mortality. The Lancet. 2014,384(9949):1215-25.

17. Worley, H. Rwanda's success in improving maternal health. Population Reference Bureau [Internet]. 2015. Disponible en: http: / / www.prb.org / Publications / Articles/2015/rwanda-maternal-health. aspx Acceso el 16 de marzo de 2018.

18. Alkema L, Chou D, Hogan D, Zhang S, Moller A, Gemmill A, et al. Global, regional, and national levels and trends in 
maternal mortality between 1990 and 2015, with scenario-based projections to 2030: a systematic analysis by the UN maternal mortality estimation inter-agency group. The Lancet. 2016;387(10017):46274. Disponible en: http://www.thelancet. com/journals/lancet/article/PIIS01406736(15)00838-7/fulltext Acceso el 16 de marzo de 2018.

19. World Health Organization (WHO). Report of the Policy Dialogue Meeting on the Nursing WorkForce, in Dialogue Meeting. Geneva: WHO; 2017:15. Disponible en: http:/ /www.who.int/hrh/ news / 2017 / NursingApril2017-2.pdf Acceso el 20 de julio de 2017.

20. National Academy for Sciences. Assessing progress on the Institute of Medicine Report The Future of Nursing, ed. Washington D.C.: TNA Press: 2016.

21. Schweitzer MC, Zoboli ELCP, Vieira MMS. Nursing challenges for universal health coverage: a systematic review. Rev Latinoam Enfermagem. 2016;24:e2676. Disponible en: http://www.scielo.br/scielo. php? script=sci_arttext\&pid $=$ S0104-116 92016000100600\&lng=en Acceso el 20 de setiembre de 2017.

22. Organización Panamericana de la Salud (OPS). Plataforma de Información en Salud de las Américas (PLISA 2017). Disponible en: http://www.paho.org/data/index. $\mathrm{php} /$ es $/$ ?option $=$ com_content\&view $=$ article\&id=515:indicadoresviz\&Itemid $=0$ Acceso el 10 de octubre de 2017.

23. Bureau of Labor Statistics, U.S. Department of Labor. Occupational Outlook Handbook; Edition, Licensed Practical and Licensed Vocational Nurses. 2016-17. Disponible en: https://www.bls.gov/ ooh/healthcare/licensed-practical-and-licensed-vocational-nurses.htm Acceso el 20 de agosto de 2017.

24. Association Canada Nursing. Nursing in Canada. Disponible en: https://www. cna-aiic.ca/en Acceso el 20 de agosto de 2017.

25. Sistema de Información Administrativa de Recursos Humanos en Enfermería de México (SIARHE). 2017. Disponible en: http://www.salud.gob.mx/unidades / cie/siarhe/?Id_URL=antecedentes Acceso el 27 de julio de 2018.

26. Ministerio de Salud Pública y Asistencia Social de Guatemala. Dirección general de recursos humanos DGRRHH/MS/PAS/ Guatemala. 2011. Disponible en: http:// www.mspas.gob.gt/index.php/institucio- nal/ unidades-departamentos / recursos-humanos Acceso el 22 de julio de 2017.

27. Instituto Nacional de Estadísticas y Censos del Ecuador. 2015. Disponible en: http:/ / www.ecuadorencifras.gob.ec/actividades-y-recursos-de-salud Acceso el 20 de julio de 2017

28. Conselho Federal de Enfermagem do Brasil (COFEN). 2017. Disponible en: http: / / www.cofen.gov.br/enfermagem-em-numeros Acceso el 10 de octubre de 2017.

29. Ministerio de Salud Presidencia de la Nación Argentina. Programa Nacional de Formación de Enfermería; Argentina 2013.

30. Departamento de Estadísticas e Información de Salud del Ministerio de Salud de Chile 2012 y Superintendencia de salud 2013. Chile, 2012.

31. Ministerio de Salud del Uruguay. Informe Recursos Humanos en Salud, SHARPS. Uruguay, 2017. Disponible en: http:// www.msp.gub.uy/sites/default/files/ Informe_Recursos_Humanos_en_Salud. pdf Acceso el 20 de julio de 2017.

32. Melo CMM, Carvalho CA, Silva LA, Leal JAL, Santos TA, Santos HS. Força de trabalho da enfermeira em serviços estaduais com gestão direta: revelando a precarização. Esc Anna Nery. 2016;20(3):e20160067. Disponible en: http://www.scielo.br/scielo.php?script $=$ sci_arttext $\&$ pid $=S 14$ 14-81452016000300211\&lng=en Acceso el 30 de enero de 2018.

33. Organization for Economic Co-operation and Development (OECD). Health workforce policies in OECD countries: right jobs, right skills, right places. Health Policy Studies OECD. 2016:187. Disponible en: http://www.keepeek.com/DigitalAsset-Management/oecd/social-issuesmigration-health / health-workforcepolicies-in-oecd-countries_9789264239517en\#.WcMnj8jyg2w Acceso el 4 de noviembre de 2017.

34. Cassiani SH, Zug KE. Promoting the advanced nursing practice role in Latin America. Rev Bras Enferm. 2014;67(5): 673-4.

35. Oldenburger D, Cassiani SHB, BryantLukosius D, Valiaitis RK, Baumann A, Pulcini J, et al. Implementation strategy for advanced practice nursing in primary health care in Latin America and the Caribbean. Rev Panam Salud Publica. 2017;41:e 40. Disponible en: http:/ / www. ncbi.nlm.nih.gov/pubmed/28614465 Acceso el 10 de octubre de 2017.
36. Chen LC, Boufford JI. Fatal flows-doctors on the move. $\mathrm{N}$ Engl J Med. 2005; 353(17):1850-2.

37. Organización Panamericana de la Salud (OPS). Llamado a la acción de Toronto: 2006-2015 hacia una década de Recursos Humanos en Salud para las Américas. Reunión Regional de los Observatorios de Recursos Humanos en Salud. Washington, 2006. Disponible en: http://www.observatoriorh.org/sites/default/files/webfiles/fulltext/OPS_desafios_toronto_2005. pdf Acceso el 4 de noviembre de 2017.

38. Naciones Unidas. Objetivos de Desarrollo Sostenible. Disponible en: http://www. un.org/sustainabledevelopment/es / Acceso el 15 de setiembre de 2017.

39. Aiken LH, Sloane DM, Bruyneel, et al Nurse staffing and education and hospital mortality in nine European coutries: a retrospective observational study. The Lancet. 2014;383:1824-30. Disponible en: http:/ / www.thelancet.com/pdfs /journals/lancet/PIIS0140-6736(13)62631-8.pdf Acceso el 25 de enero de 2018.

40. Aiken LH, Clarke SP, Cheung RB, Sloane DM, Silber JH. Educational levels of hospital nurses and surgical patient mortality. JAMA. 2003;290(12):1617-23. Disponible en: https://www.ncbi.nlm.nih.gov/pmc/ articles/PMC3077115/ Acceso el 31 de enero de 2018.

41. Agencia Informativa Latinoamericana. Migración de personal de enfermería provoca crisis en Jamaica. Nota Informativa. Disponible en: http://prensa-latina.cu/ index.php? o $=\mathrm{rn} \& \mathrm{id}=59710 \& \mathrm{SEO}=$ migracion-de-personal-de-enfermeriaprovoca-crisis-en-jamaica Acceso el 10 de octubre de 2017.

42. Organización Mundial de la Salud (OMS). Manual de seguimiento y evaluación de los recursos humanos para la salud: con aplicaciones especiales para los países de ingresos bajos y medianos [Internet]. OMS; 2009:212. Disponible en: http:// apps.who.int/iris/bitstream/10665/ 44187/1/9789243547701_spa.pdf Acceso el 16 de marzo de 2018.

Manuscrito recibido el 13 de noviembre de 2017. Aceptado para su publicación, tras revisión, el 20 de marzo de 2018. 
SUMMARY

Distribution of the nursing workforce in the Region of the Americas

Keywords Labor force; human resources; nursing staff; Americas.
Objective. Describe the distribution of the nursing workforce in countries of the Region of the Americas and the relation between the number of human resources in nursing and maternal mortality ratios.

Methods. Descriptive and exploratory analysis of 27 countries of the Region. The variables in the study were the proportion of health professionals per country and subregion; professional category; and nurse-to-physician ratio. The maternal mortality ratio was used as an overall indicator of the health of the population to analyze its relationship to the number of nurses. Frequency distribution and density of human resources in nursing were analyzed per country and subregion.

Results. The distribution of nursing personnel was heterogeneous. Some countries had more than 80 nurses per 10000 population and others had fewer than five professionals per 10000 population. In $34.1 \%$ of the countries, the nurse-to-physician ratio was less than 1. Differences in the distribution of nursing personnel were observed between regions and subregions, and within countries.

Conclusions. In several countries, the number of nurses per capita was less than expected. The majority of the countries showed a significantly lower proportion of licensed nurses with respect to technical and auxiliary personnel. It is necessary to implement initiatives to increase the number of licensed nurses throughout the Region.

RESUMO Objetivo. Descrever a distribuição de pessoal de enfermagem nos países da Região das Américas e estabelecer a relação do número de profissionais de enfermagem com o índice de mortalidade materna.

Distribuição de pessoal de enfermagem na Região das Américas

Métodos. Estudo descritivo e exploratório conduzido em 27 países da Região das Américas. As variáveis estudadas foram proporção de profissionais por país e subregião, categoria profissional e relação enfermeiro-médico. $\mathrm{O}$ índice de mortalidade materna, por ser um indicador geral de saúde da população, foi usado para analisar a relação com o aspecto quantitativo de enfermeiros. Foram analisadas a distribuição de frequências e a densidade dos profissionais de enfermagem por país e sub-região.

Resultados. Verificou-se uma distribuição heterogênea do pessoal de enfermagem, com alguns países com mais de 80 enfermeiros por 10 mil habitantes e outros com menos de cinco profissionais por 10 mil habitantes. Observou-se uma relação enfermeiro-médico inferior a um em 34,1\% dos países. Houve diferenças na distribuição de pessoal de enfermagem por região, sub-região e dentro do próprio país.

Conclusões. Em vários países da Região das Américas, o número de enfermeiros por habitantes é menor que o esperado. A maioria dos países tem uma defasagem considerável na razão do número de enfermeiros licenciados em relação ao pessoal de enfermagem técnico e auxiliar. É necessário implementar iniciativas para aumentar o número de enfermeiros licenciados em toda a Região.

Palavras-chave Força de trabalho; recursos humanos; recursos humanos de enfermagem; Américas. 